\title{
Notas sobre o Behaviorismo de Ontem e de Hoje
}

\author{
Remarks on Former and Current Behaviorism
}

\author{
Emmanuel Zagury Tourinho* \\ Universidade Federal do Pará, Belém, Brasil
}

\begin{abstract}
Resumo
Este artigo busca oferecer algumas referências históricas sobre as origens e desenvolvimentos da Psicologia behaviorista. $\mathrm{O}$ artigo parte das contribuições de Watson, destacando seu afastamento do modo causal selecionista, inaugurado na Psicologia por Thorndike. Em seguida, discute alguns desenvolvimentos do behaviorismo sob a liderança de Skinner, apontando que seus avanços resultam em grande medida do retorno ao selecionismo. $\mathrm{O}$ artigo argumenta, por fim, que ao final do século XX a abordagem inaugurada por Skinner estende-se a em muitas direções, configurando a análise do comportamento como um sistema multidimensional. O artigo conclui com a indicação de que uma visão histórica do processo de investigação comportamental oferece um quadro mais preciso do que essa abordagem tem representado enquanto sistema na Psicologia.
\end{abstract}

Palavras-chave: Behaviorismo; Behaviorismo radical; Análise do comportamento; História da Psicologia.

\begin{abstract}
This paper aims at offering some historical references concerning the origins and development of behaviorist Psychology. It begins with Watson's contributions and highlights his detachment from the causal mode of selection by consequence started in Psychology by Thorndike. Then, the paper discusses some developments of behaviorism under the leadership of Skinner and argues that some achievements have largely resulted from a recovery of selectionism. Finally, it contends that, by the end of the $20^{\text {th }}$ century, the approach started by Skinner extended to new directions, engendering behavior analysis as a multidimensional system. The paper concludes by pointing out that a historical view of the process of behavioral investigation provides a more precise frame of what this approach has represented as a psychological system.

Keywords: Behaviorism; Radical Behaviorism; Behavior Analysis; History of Psychology.
\end{abstract}

Os manuais de Psicologia apresentam o behaviorismo como uma escola de pensamento na Psicologia, inaugurada por John B. Watson (1878-1958), com seu manifesto de 1913 (Watson, 1913/1994). Essencialmente, essa escola se definia pela proposição do comportamento como objeto de estudos da Psicologia, pela idéia de continuidade entre as espécies e pela adoção dos métodos de investigação das ciências naturais: observação e experimentação. O primeiro parágrafo do manifesto de Watson afirmava:

A Psicologia, como um behaviorista a vê constitui um ramo puramente experimental da ciência natural. Seu objetivo teórico é a previsão e o controle do com-

\footnotetext{
* Endereço para correspondência: Universidade Federal do Pará, Rua Augusto Corrêa, 01, Guamá, Belém, PA, Brasil, CEP 66075-110. E-mail: eztourinho@gmail.com $\mathrm{O}$ autor agradece a Simone Neno pelos comentários a uma versão preliminar do texto.

Trabalho parcialmente financiado pelo Conselho Nacional de Desenvolvimento Científico e Tecnológico (Processos 304116/2007-6 e 480727/2007-4). Partes deste trabalho foram apresentadas na $59^{\mathrm{a}}$ Reunião Anual da Sociedade Brasileira para o Progresso da Ciência (SBPC), Belém, 2007.
}

portamento ... Em seu esforço para alcançar uma concepção unitária da resposta animal, o behaviorista não reconhece qualquer linha divisória entre o homem e o bruto. O comportamento humano, com todo o seu refinamento e complexidade constitui apenas uma parte do programa completo de investigação do behaviorista (p. 248).

No presente artigo, pretende-se apontar algumas feições que o behaviorismo veio a assumir ao longo do século XX, indicando que a referência a Watson constitui um passo importante, porém atualmente insuficiente para uma compreensão do que essa abordagem tem representado no cenário da Psicologia, tanto do ponto de vista histórico, quanto em termos de seus impactos nas formulações contemporâneas dos chamados problemas psicológicos. Algumas das mais significativas contribuições do behaviorismo têm sido, e continuam sendo, a concepção relacional do comportamento humano, a visão selecionista da causação do comportamento, a interpretação funcional, não representacional, da linguagem e dos processos lingüísticos e a proposição de que os fenômenos psicológicos são relações do homem com o mundo, que podem ser abordadas com os conceitos de uma ciência do comportamento. Essas contribuições, 
como argumentado adiante, derivam substancialmente de autores que antecederam ou sucederam Watson na proposição de uma ciência do comportamento.

\section{O Comportamento como Objeto da Psicologia}

Watson não deu início à investigação do comportamento em Psicologia. Mais de uma década antes de seu manifesto (Watson, 1913/1994), o comportamento animal já era estudado experimentalmente por pesquisadores que se ocupavam de questões então consideradas afeitas ao campo psicológico. O mais notório exemplo da psicologia comportamental do século XIX foi Edward Lee Thorndike (1874-1949), que estudou a aprendizagem em humanos e não humanos e formulou a lei do efeito. Se alguma dúvida houver sobre a pertinência da pesquisa de Thorndike aos assuntos da Psicologia, basta considerar o título da monografia de 1898 na qual já estava implícita (cf. Catania, 1999) a Lei do Efeito: A Inteligência Animal (Thorndike, 1898/1998). Nessa monografia, Thorndike fazia referência à "vida mental dos animais" (p. 1125) e buscava examinar seus processos de "associação e hábitos". O artigo sumarizava seus experimentos de então, com gatos em caixas-problema, em que eram colocados em privação de alimento e apenas quando emitiam respostas específicas (pisar em uma plataforma, puxar uma corda, ou pressionar uma barra) ganhavam acesso ao alimento. Uma referência ao que mais tarde seria apresentado como a lei do efeito aparecia na conclusão do texto: as associações "significam simplesmente a conexão de um determinado ato com uma determinada situação e o prazer resultante" (p. 1127).

Também em 1898, Thorndike já postulava a necessidade de "experimentos cruciais, planejados cuidadosamente", que poderiam informar corretamente sobre o comportamento animal, ou melhor, poderiam "não apenas dizer precisamente o que eles fazem e prover a tão necessária informação sobre como o fazem, mas também informar-nos sobre o que sentem enquanto agem" (1898/ 1998, p. 1126). Segundo Hearst (1999), os estudos de Thorndike em 1898 teriam sido reconhecidos pelo próprio e por outros autores (Pavlov incluído) como "a primeira aplicação ampla e deliberada do método experimental à aprendizagem animal" (p. 441).

No que concerne à lei do efeito, o que é tido como uma versão final apareceu apenas em 1911, mas, como aponta Catania (1999), uma primeira versão formal já era encontrada em um texto de 1901, ainda que nesse último caso a referência não fosse à seleção do comportamento, mas apenas à seleção de conexões neurais:

$\mathrm{O}$ aumento gradual no sucesso significa um fortalecimento gradual de um conjunto de conexões nervosas e um enfraquecimento gradual de outras. Este método de aprendizagem pode ser chamado de método de ensaio e erro, ou de tentativa e sucesso (Thorndike, 1901, citado por Catania, 1999, p. 427).
A seleção do comportamento pelas conseqüências aparece com clareza na versão final da lei do efeito, publicada em 1911, dois anos ainda antes do manifesto watsoniano. No lugar de ater-se a pormenores da topografia da resposta, a propriedades como latência ou força da resposta, a lei do efeito sugeria que o cientista do comportamento deveria voltar sua atenção para mudanças na probabilidade da resposta, como função de mudanças na "satisfação" do organismo produzida por sua ação.

Das várias respostas à mesma situação, aquelas que são acompanhadas ou seguidas de perto pela satisfação do animal serão, mantidas iguais as outras coisas, mais firmemente conectadas com a situação, de modo que quando [a situação] voltar a ocorrer será mais provável que voltem a ocorrer; aquelas que são acompanhadas ou seguidas de perto pelo desconforto do animal terão, mantidas iguais as outras coisas, suas conexões com aquela situação enfraquecidas, de modo que quando [a situação] voltar a ocorrer será menos provável que voltem a ocorrer. Quanto maior a satisfação ou o desconforto, maior o fortalecimento ou enfraquecimento do vínculo (Thorndike, 1911/2007, p. 118).

Um aspecto desta formulação da lei do efeito foi alvo de críticas freqüentes por parte de psicólogos comportamentais: a referência à satisfação ou estados internos do organismo como parte da explicação para o comportamento, o que representaria uma volta a visões internalistas na Psicologia. A elaboração de Thorndike, porém, assemelha-se em muito à proposição de Skinner (1986/1987b), de que o reforço tem dois efeitos: um aumento na probabilidade da resposta e uma sensação de prazer.

As pesquisas de Thorndike com animais também anteciparam o desenvolvimento de aparatos experimentais que possibilitaram o uso de medidas objetivas de mudanças comportamentais. Com suas caixas-problema para estudos com gatos, Thorndike registrava mudanças no tempo que o animal levava para sair da caixa, assim como observava a redução na variabilidade da resposta. A mudança na curva do tempo da resposta representava, para Thorndike, a "taxa de aprendizagem" (Chance, 1999, p. 436).

Com Thorndike, a influência de Darwin sobre os psicólogos experimentais vai além da noção de continuidade entre as espécies. A lei do efeito também antecipava uma das principais realizações da ciência do comportamento ao longo do século XX: a superação do mecanicismo e a elaboração do selecionismo como modo causal explicativo do comportamento. Como Catania (1999) aponta:

é interessante [notar] que o papel da seleção por conseqüências recebeu um tratamento explícito somente relativamente tarde no trabalho de Skinner, quase meio século após Thorndike ter virtualmente iniciado sua carreira tratando a aprendizagem em termos de seleção (p. 427). 
No modo causal mecanicista, o responder do organismo é explicado por eventos que o antecedem e o determinam; as relações comportamentais são unidirecionais, invariáveis, e os eventos que dela participam independem uns dos outros. A lógica mecanicista conduz o cientista psicólogo, portanto, a olhar o organismo como um ser reativo; e aos eventos que participam de relações comportamentais (estímulos e respostas) como entidades reais e anteriores ao contexto das relações.

Em uma direção oposta, no selecionismo, o responder é explicado por suas funções na relação com conseqüências que produz. Nesse caso, não há um ambiente anterior e independente que produza o responder do organismo, mas um ambiente produzido pelo próprio organismo, dependente de suas ações, e que só se constitui efetivamente como ambiente (isto é, deixa de ser uma parte indiferenciada do mundo que cerca o organismo) quando adquire alguma função comportamental. Isto é, o mundo que afeta o responder do organismo é produzido pelo próprio organismo, não existe antes e independentemente de sua ação (Sério, 1997). Por outro lado, mudanças ambientais produzidas pelo responder de um organismo afetam suas ações futuras (quando as afetam) apenas probabilisticamente, aumentam ou diminuem probabilidades de resposta, visto que são parte de um contexto mais amplo de variáveis relevantes. No modo causal selecionista, portanto, não é possível falar de relações invariáveis (o probabilismo implica variabilidade, em menor ou maior grau), relações lineares (estímulos e respostas determinam-se mutuamente), ou de entidades pré-existentes ao contexto de relações comportamentais. Respostas e estímulos (incluindo estímulos reforçadores ou punidores) são funções que ocorrências do organismo e do ambiente adquirem, e definem-se apenas mutuamente, em um contexto de relação organismo-ambiente. Como aponta Dinsmoor (2004), um estímulo é "meramente um elemento detectável no ambiente circundante, capaz de exercer uma variedade de funções" (p. 311). No lugar do determinismo (ou do determinismo absoluto, dependendo da terminologia preferida), é necessário falar de probabilismo (ou determinismo probabilístico). Além disso, a abordagem requerida para o exame de relações comportamentais será necessariamente funcionalista, visto que está-se partindo de uma noção de fenômeno comportamental como relações de dependência funcional entre estímulos e respostas. Adiante, o tema do selecionismo será retomado. Aqui, cabe ressaltar a importância do advento do modo causal selecionista na ciência do comportamento e o fato de que é com Thorndike que essa perspectiva é inaugurada.

Curiosamente, o estudo das relações resposta-consequiência por muito tempo foi negligenciado e, na melhor das hipóteses, disputou a atenção de behavioristas com o estudo de relações reflexas ou respondentes, que havia se difundido com o trabalho de Ivan Petrovich Pavlov (1849-1936). Pavlov não era psicólogo, mas físiologista, interessado principalmente no processo digestivo. Em 1904, recebeu o Prêmio Nobel de Fisiologia e Medicina por estudos sobre a digestão. Mais tarde, sua indicação de que as respostas de um organismo podem vir a ser eliciadas por estímulos condicionados pareceu ser um bom caminho para o estudo da aprendizagem em geral e um bom ponto de partida para o estabelecimento de um programa amplo de pesquisas (cf. Dinsmoor, 2004; Skinner, 1931/1961; Todorov, 2004), o que a lei do efeito não havia realizado.

Nos trabalhos de Watson (e.g., 1930/1970), a referência ao reflexo constituía o núcleo da explicação do comportamento em geral, inclusive do comportamento humano complexo. Este último se explicava não pela referência a processos adicionais ao reflexo, mas pela sucessão de processos de condicionamento respondente. Essa perspectiva representava uma extensão de princípios e procedimentos da investigação pavloviana, isto é, o condicionamento respondente, do campo fisiológico para o fenômeno do comportamento. Como psicólogo, porém, Watson estava interessado no comportamento não de sistemas orgânicos particulares (como o sistema glandular), mas do organismo como um todo (isto é, como a ação integrada dos vários sistemas orgânicos). Essa abordagem valia tanto para respostas simples, como pressionar um botão, quanto para fenômenos complexos, como o pensamento. $\mathrm{O}$ foco no comportamento do organismo como um todo, também implicava estudar o organismo intacto, sem as manipulações típicas dos estudos fiológicos de Pavlov, que justificavam invadir o organismo com instrumentos para medir variações em um sistema ou subsistema orgânico. Posteriormente, a idéia de olhar para o comportamento do organismo como um todo e sem invasão instrumental mostrou-se central para a proposição skinneriana (cf. Skinner, 1931/1961) de que o reflexo deveria ser tomado como objeto de estudo da Psicologia.

A originalidade e repercussão do trabalho de Watson derivaram provavelmente menos da proposição do estudo experimental do comportamento, do que da difusão da noção de que aquele programa de pesquisas representava o curso possível para o estabelecimento da Psicologia como disciplina científica. A desqualificação da introspecção como método veio a ser, desse ponto de vista, apenas $u m$ aspecto de um movimento mais amplo, que consistia da desqualificação de programas diversos de investigação que constituíam os horizontes da Psicologia no início do século XX (da psicologia da consciência à psicanálise freudiana). Note-se, ainda, que os princípios derivados do estudo do condicionamento mantiveram sua validade e utilidade no ambiente da pesquisa e da intervenção comportamental, mesmo após deixarem de ocupar lugar central na explicação e na configuração de programas de investigação científica do comportamento. 


\section{B. F. Skinner e a Inauguração da Análise do Comportamento}

B. F. Skinner (1904-1990) foi um legítimo sucessor de Watson, ao mesmo tempo em que conferiu ao movimento behaviorista impressões próprias, alargou enormemente os horizontes da investigação científica do comportamento e deu origem a uma extensa comunidade científica reunida em torno de programas de investigação por ele inaugurados.

Seguindo o percurso de Watson, Skinner iniciou sua carreira estudando o reflexo (cf. Skinner, 1931/1961), mas já no início desse estudo apareceram as marcas de seu projeto. Interessava-o não simplesmente a possibilidade do condicionamento, ou sua suficiência para explicar a aprendizagem, mas principalmente a variabilidade do reflexo (cf. Sério, 1990; Skinner, 1938). Skinner buscava as "leis da variabilidade", o que, de certa forma, já começava a afastá-lo da lógica mecanicista, mesmo tomando o reflexo como objeto de estudos. As relações estímulo antecedente-resposta, no lugar de relações invariáveis, passaram a ser vistas como dependentes de "terceiras variáveis", inicialmente formuladas com a referência ao condicionamento, ao drive (motivação) e à emoção, conceitos com os quais Skinner (1987a) alega que estaria ainda abordando condições ambientais externas ao organismo.

É com a investigação de relações operantes, porém, a partir do final da década de 30 , que o sistema skinneriano começou a assumir seus contornos definitivos, dando origem ao programa de pesquisas que viria a exercer grande impacto na Psicologia experimental das três décadas seguintes. A proposição do operante, em 1937, e, principalmente, no livro O Comportamento dos Organismos (Skinner, 1938), restaurava o interesse pelas conseqüências do comportamento, ou melhor, o reconhecimento de suas funções causais, como assinaladas por Thorndike quarenta anos antes.

A relação entre a proposição do operante e o trabalho de Thorndike acabou sendo alvo de uma controvérsia, devido ao fato de que o livro de Skinner não atribuía o crédito devido à lei do efeito. Em 1939, Hilgard (1939/ 1988) publicou uma crítica ao Comportamento dos Organismos (Skinner, 1938), por essa razão (no livro todo, havia uma única referência, bastante genérica, à lei do efeito). Após a publicação da crítica, Skinner escreveu uma carta a Thorndike, na qual tentava justificar-se, porém em termos não menos problemáticos:

A revisão de Hilgard para o meu livro . . . fêz-me lembrar o quanto deixei de reconhecer do seu trabalho na mesma direção. Investigando minha alma para descobrir porque o reconhecimento nunca foi feito, cheguei a apenas o seguinte: 1) eu nunca vi um "sistema" anunciado e promovido com o seu nome; e

2) eu pareço ter identificado o seu ponto de vista com a concepção psicológica moderna como um todo. Sempre foi óbvio [para mim] que eu estava sim- plesmente dando continuidade a seus experimentos com caixas-problema, mas nunca me ocorreu de lembrar meus leitores desse fato. Eu não sei porque menciono isso, pois não posso imaginar que isso o incomode minimamente. (Skinner, 1939, citado por Hearst, 1999, p. 445).

A resposta de Thorndike foi de uma elegância absoluta: "Eu fico mais satisfeito de ter sido útil para trabalhadores como você, do que se tivesse fundado uma 'escola'" (Thorndike, 1939, citado por Hearst, 1999, p. 445).

O outro lado dessa controvérsia é que apenas o trabalho de Skinner deu origem a um programa amplo e diversificado de investigações sobre a seleção do comportamento por suas conseqüências, sobretudo com a sistematização do que veio a ser designado como esquemas de reforço (Ferster \& Skinner, 1957), que definem parâmetros ao longo dos quais variações nas relações resposta-conseqüência afetam o responder geral do organismo. Além disso, ainda que muito tardiamente, considerando a antecipação de Thorndike, é apenas com Skinner que o selecionismo como modo causal encontra uma elaboração refinada na ciência do comportamento; uma elaboração que articula as relações entre filogênese, ontogênese e cultura como níveis de seleção do comportamento, e que operam ora em uma mesma direção, ora em direções conflitantes, sobrepondo-se uns aos outros (cf. Skinner, 1990). Foi a elaboração skinneriana que definitivamente afastou o mecanicismo, assinalando a bidirecionalidade, variabilidade e dependência mútua das relações entre respostas e estímulos, e o caráter probabilístico da determinação das primeiras, uma perspectiva ainda não inteiramente explorada na própria ciência do comportamento. Timberlake (1993) assinala a originalidade da abordagem skinneriana:

Embora a maior parte dos behavioristas tenha seguido a liderança de Thorndike, postulando uma ligação causal simples entre reforçador e resposta, Skinner (1938) percorreu um caminho relativamente diferente. Ele argumentou que o reforçamento não era um exemplo de simples causa e efeito, mas uma forma de seleção por conseqüências, um modo causal único e característico de seres vivos (p. 106).

O horizonte do projeto skinneriano sempre foi o comportamento humano. Desde que ingressou no curso de doutorado em Psicologia, em Harvard, em 1928, Skinner era movido pela expectativa de oferecer uma resposta para questões de ordem epistemológica. Uma ciência do comportamento deveria ser capaz de explicar o comportamento humano em geral, inclusive o comportamento verbal do cientista. Contribuiu, para essa visão, a leitura que Skinner havia realizado de trabalhos de Russell (cf. Smith, 1989).

Skinner trabalhava em uma análise funcional para o comportamento verbal desde pelo menos 1934 (cf. Skinner, 1979), e uma versão havia sido apresentada no Simpósio sobre Operacionismo, em 1945 (Skinner, 
1945). Todavia, o livro Comportamento Verbal (Skinner, 1957/1992) foi publicado somente em 1957. Muito mais tardiamente, ainda, apenas na década de 80 , os analistas do comportamento começaram a se dedicar a programas de pesquisa sobre o comportamento verbal.

A proposta de Skinner (e.g., 1945, 1957/1992) subverte radicalmente muitas concepções tradicionais sobre a linguagem: no lugar de algo armazenado, a linguagem é comportamento, comportamento verbal; no lugar de idéias ou imagens mentais, os significados encontram-se nas contingências de reforço; no lugar de representar uma realidade independente, nossos conceitos definem modos particulares de interação com parcelas da realidade; no lugar de um acesso privilegiado à consciência, nossas autodescrições limitam-se pelas práticas de uma comunidade verbal.

Segundo Hayes, Barnes-Holmes e Roche (2001), na construção de uma abordagem funcional para a linguagem Skinner teria usufruído da interlocução com J. R. Kantor (1888-1984), com quem trabalhou por um curto período. Como Skinner, Kantor teve papel destacado na construção do behaviorismo ao longo do século XX, e é muitas vezes descrito como aquele que produziu a mais consistente fundamentação filosófica para uma concepção funcional-comportamental, anti-mentalista, dos fenômenos psicológicos, justificando a interpretação de muitos acerca da larga compatibilidade de seu sistema de pensamento, o interbehaviorismo, com a análise do comportamento (cf. Moore, 1987; Morris, 1984). Kantor era chefe do Departamento de Psicologia na Indiana University, quando Skinner lá permaneceu por dois anos, de 1945 a 1947. A influência que exerceu sobre o pensamento skinneriano ainda está por ser plenamente conhecida. Sabe-se que foi Kantor quem convenceu Skinner acerca da impropriedade do conceito de drive. Mais precisamente, o conceito de drive era apontado por Kantor como um exemplo das "assombrações" ("spooks") que tomavam conta da Psicologia não científica (cf. Morris, Higgins, \& Bickel, 1982; Verplanck, 1995).

Dentre as contribuições de Kantor para o behaviorismo contemporâneo, encontra-se sua ênfase na caracterização do objeto da Psicologia como sendo a interação organismo-ambiente, afastando-se de qualquer viés reducionista e destacando o caráter relacional do fenômeno comportamental. Kantor elaborou uma crítica original (já na década de 20 do século passado) ao internalismo organicista, tão popular, nos tempos atuais, por força de interpretações equivocadas dos achados das neurociências. Também na década de 20, Kantor (1922) já apontava a insuficiência do conceito de reflexo e da idéia de integração de vários reflexos para explicar a varibilidade $\mathrm{e}$ complexidade do comportamento humano.

Bem mais tarde, Kantor (1970) avançou na discussão dos modos como a pesquisa do condicionamento respondente acabou impondo limitações a todo o programa de pesquisa da análise experimental do comportamento, por exemplo levando-a a ocupar-se excessivamente do condicionamento, agora operante, e da investigação $e x-$ perimental em ambientes controlados. Essa análise de Kantor foi apresentada em um evento da divisão 25 (Análise Experimental do Comportamento) da American Psychological Association (APA) e sua publicação posterior, no Journal of the Experimental Analysis of Behavior (JEAB), foi motivo de tensionamento entre kantorianos e skinnerianos. Apesar disso, um grupo de interesse especial foi criado na Association for Behavior Analysis (ABA), para tratar do interbehaviorismo (o sistema kantoriano). Desde o artigo de Kantor de 1970, Skinner vinha evitando créditos ao pensamento kantoriano (cf. Verplanck, 1995) e, em 1988 (Skinner, 1988), reagiu à constituição daquele grupo de interesse especial na $\mathrm{ABA}$, em uma publicação da própria $\mathrm{ABA}$, reclamando que passáros "cucos" (na gíria norte-americana, "malucos") estavam "colocando ovos em seu (de Skinner) ninho" (Verplanck, 1995). É de Kantor, por último, a mais antidualista elaboração do problema da subjetividade, que, em conjunto com as contribuições de Skinner, permite a formulação de uma interpretação bastante avançada da questão (cf. Tourinho, 2004).

Voltando à interpretação skinneriana para a linguagem, pode-se dizer que com ela a psicologia behaviorista passou a dispor, finalmente, de uma abordagem que, fazendo uso de conceitos científicos, refutou toda visão representacional da linguagem e do conhecimento. Em vez de tentar fundamentar o conhecimento objetivo, Skinner entendia que a objetividade é uma questão de contingências sócio-verbais. $\mathrm{O}$ valor das descrições científicas, seu caráter de verdade, depende de suas funções no controle de uma interação produtiva com a realidade, não da apreensão de uma essência qualquer, inacessível às mentes menos disciplinadas. Isso vale para as próprias descrições ou enunciados de uma ciência do comportamento. A medida de seu valor é a medida do quanto auxilia o cientista e o profissional psicólogo a lidar com os fenômenos comportamentais. Basta comparar essa perspectiva com a adesão ao positivismo lógico de alguns behavioristas anteriores a Skinner (cf. Smith, 1989), para ter a medida do que representou a elaboração de uma interpretação comportamental-funcional para a linguagem.

Os impactos de uma abordagem funcional para o comportamento verbal estendem-se, ainda, a todo um vasto espectro de problemas dos quais a Psicologia tem tradicionalmente se ocupado, incluindo aqueles relativos a cognições e emoções. Infelizmente, porém, novos avanços nesse terreno não aconteceram até a década de 80 , quando o cognitivismo, tendo se ocupado desses temas por duas décadas, inclusive apoiado em modelos de investigação e intervenção originalmente comportamentais, já se tornara dominante no cenário acadêmico e profissional da Psicologia norte-americana.

Os dois programas de pesquisa que de modo mais sistemático e inovador têm se ocupado de relações comportamentais verbais na análise do comportamento são 
aqueles que se voltam para "relações de equivalência", sob a liderança de Murray Sidman (cf. Sidman, 1994), e para a formação de "quadros relacionais", sob a liderança de Steven C. Hayes (cf. Hayes et al., 2001). Esses programas têm produzido recursos empíricos e analíticos para o exame de processos lingüísticos ou verbais, por meio dos quais eventos arbitrariamente relacionados, de diferentes modos, adquirem funções novas para o responder de um indivíduo, dando origem a repertórios complexos e variados ("operantes relacionais", na designação de Hayes), que não resultam de condicionamentos específicos, ou da aprendizagem direta. Em ambos os casos, trata-se de programas ao mesmo tempo heurísticos e controversos; que têm impulsionado a investigação do comportamento humano complexo, mas cuja compatibilidade com o sistema explicativo skinneriano é freqüentemente colocada em discussão (e.g., Burgos, 2003; Hall \& Chase, 1991; Palmer, 2004; Ribeiro, 1995).

\section{A Análise do Comportamento Contemporânea}

A visão não representacional da linguagem, postulada pela Análise do Comportamento, está em acordo com muitos sistemas de pensamento que, ao longo do século $\mathrm{XX}$ foram construídos na filosofia e nas ciências humanas. Sistemas de pensamento que não são designados como behavioristas, principalmente porque não têm raízes históricas na psicologia behaviorista, em particular na psicologia behaviorista norte-americana, mas que, em essência, partilham noções de uma visão antidualista dos fenômenos humanos e anti-representacional do conhecimento e da linguagem; sistemas, portanto, que também conflitam com certas concepções modernas acerca do homem, suas faculdades enquanto indivíduo e suas relações com o mundo físico e social.

Há vários exemplos desses sistemas não behavioristas, mas muito compativeis com o behaviorismo, por exemplo, a interpretação de Wittgenstein (1953/1988) para a linguagem como forma de ação no mundo, a discussão de Rorty (1993) acerca da objetividade como solidariedade, a crítica sociológica de Elias (1994) ao conceito moderno de indivíduo e o materialismo cultural de Harris (2007) na análise da evolução das culturas.

Voltando ao próprio behaviorismo, ou à comunidade científica que se estruturou ao longo do século XX, sob a liderança, principalmente, do trabalho de Skinner, mudanças substanciais aconteceram especialmente no que diz respeito a uma maior diversificação das produções. A investigação básica do comportamento conhecida como análise experimental do comportamento por muitos anos foi, e continua sendo, o tipo de produção mais freqüente e mais estruturada, no sentido de que representa o esforço de grupos numerosos de pesquisadores, organizados em torno de programas de pesquisas razoavelmente compartilhados e bem definidos. Outros tipos de produção, no entanto, floresceram e passaram a aglutinar analistas do comportamento, dando origem a novos programas de pesquisa e aplicação, a novas revistas científicas e a novas categorizações de atividades em eventos científicos (cf. Tourinho \& Sério, 2010).

A essas mudanças estão associadas diferentes denominações do campo de saber que representa a tradição de pensamento inaugurada por Skinner, que se tornaram dominantes em diferentes momentos históricos: behavioristas, behavioristas radicais, analistas experimentais do comportamento e modificadores de comportamento, por exemplo. Atualmente, esse campo de saber tem sido mais sistematicamente denominado de análise do comportamento. Mais importante do que isso, tem-se observado um esforço para caracterizar a análise do comportamento como um campo de saber multidimensional. Ao lado da investigação básica, usualmente experimental, dos processos comportamentais, entendem-se como produções constitutivas do campo analítico-comportamental trabalhos teóricos, conceituais, históricos, filosóficos, pesquisa aplicada e aplicações profissionais da análise do comportamento.

Pode-se pensar as diferentes produções analíticocomportamentais como reguladas umas pelas outras e mais ou menos aproximadas de três vértices de um triângulo epistêmico, constituídos por o que podemos chamar de produções reflexivas, investigação básica e aplicações da análise do comportamento. Essa caracterização toma como referência o próprio histórico de constituição da Psicologia, inicialmente como um campo reflexivo, no interior dos debates epistemológicos e, mais tarde, como ciência e como profissão.

A rigor, nenhuma produção em análise do comportamento pertence a apenas um vértice, posto que não se faz indiferente ao que acontece no conjunto das realizações da área. Uma produção pode aproximar-se muito mais de uma área, isto é, pode ser mais reflexiva, mais aplicada, ou mais básica, nesse caso atendendo mais claramente um tipo de demanda que conforma o campo da Psicologia. Mas a relação com outros tipos de produção e outras demandas da cultura também estão presentes.

Assim, produções que se aproximam mais do vértice da investigação básica são aquelas que dialogam prioritariamente com a demanda por um conhecimento cientificamente validado sobre as regularidades dos fenômenos comportamentais. Produções aplicadas consistem dos usos do saber analítico-comportamental em contextos de intervenção profissional com vistas à solução de problemas humanos. Nesse caso, os problemas de que os analistas aplicados se ocupam são, em geral, aqueles que se relacionam com o processo de individualização no mundo moderno (por exemplo, no campo da educação, do trabalho e da saúde), para os quais o trabalho do psicólogo é usualmente requisitado. Produções reflexivas consistem de investigações metacientíficas, do tipo conceitual, teórica, histórica, filosófica, etc. Uma característica especial das produções reflexivas é que elas têm criado áreas de interlocução da análise do comportamento com outras disciplinas, o que é especialmente relevante 
considerando-se que, com o declínio do pensamento comportamental e ascensão do cognitivismo, os antigos "behavioristas" passaram a experimentar um certo isolamento no cenário acadêmico.

Se é verdade que, contrariando algumas sentenças, o behaviorismo não está morto, e realmente não está, como o reconhecem mesmo psicólogos cognitivistas (cf. Roediger, 2004), também é verdade que ao longo do século $\mathrm{XX}$ foi perdendo terreno no diálogo com áreas diversas da cultura, o que repercutiu de muitos modos em seu status e alcance no mundo acadêmico. A popularidade do behaviorismo na universidade e na sociedade nunca foi excepcional, é bom que se diga, mas certamente sofreu um declínio na segunda metade do século XX. As razões para isso foram tanto internas (como o caráter cada vez mais microscópico das análises oferecidas para o comportamento - cf. Roediger, 2004), quanto externas (como o advento de programas cognitivistas que sugeriam ser possível erigir uma psicologia ao mesmo tempo científica e interessada na mente).

A atual diversificação de produções em análise do comportamento, por outro lado, talvez represente um movimento original, de vitalidade da área, revelando sua capacidade de intervir nos diferentes ambientes de construção da Psicologia e sua disposição para dialogar com sistemas de pensamento em outras áreas de conhecimento.

$\mathrm{Na}$ origem, o enfoque comportamental representou um abandono não apenas do método da introspecção, mas do interesse pelas questões relacionadas às cognições e emoções, reconhecendo-as como mentais e para além dos horizontes de uma ciência. Contribuiu para essa posição a adesão de alguns psicólogos comportamentais a preceitos do positivismo lógico e de doutrinas a ele associadas. No entanto, esse não foi o percurso da análise do comportamento. Uma das contribuições mais representativas da análise do comportamento consiste exatamente em dissolver aquelas suspeitas sobre uma natureza especial dos fenômenos subjetivos, em indicar que reproduzem uma lógica dualista cartesiana, em sustentar a possibilidade de submetê-la ao inquérito científico e em oferecer uma interpretação relacional para os problemas que representam.

A abordagem comportamental para a questão da subjetividade tem basicamente duas direções, que podem ser brevemente indicadas. Uma primeira direção é lingüística. Se nossos conceitos, incluindo os conceitos emocionais, são relações verbais, isto é, relações mediadas pelos outros, relações que dependem de contingências sociais verbais, possuem, então, invariavelmente, uma base pública, não podem descrever algo estritamente pessoal, interno, ou inacessível. Quando um indivíduo se descreve feliz, a base para o seu relato não pode ser uma ocorrência íntima, privada, ou interna; diferente disso, será necessariamente a mesma base para que ele atribua felicidade a outra pessoa. Neste ponto, a análise do comportamento segue uma linha de argumentação semelhante à encontrada na obra Investigações Filosóficas, de Wittgenstein (1953/1988), e no livro O Conceito de Mente, de Ryle (1949/1984). Para a análise do comportamento (cf. Skinner, 1945), de uma abordagem funcional para a linguagem decorre, necessariamente, o reconhecimento de que os conceitos não representam essências, de qualquer natureza, que transcendam as condições de interação entre os membros de uma comunidade verbal.

O behaviorismo skinneriano acrescentará a esse ponto de vista uma posição também encontrada em Wittgenstein (1953/1988), mas não em Ryle (1949/1984): o reconhecimento de que, de modo circunstancial e parcial, uma resposta verbal pode ser emitida sob controle de um evento que não está acessível à observação pública. Por exemplo, apesar de sua base pública, uma resposta verbal do tipo "estou feliz" pode eventualmente ser emitida sob controle de um evento inacessível aos outros (por exemplo, uma estimulação gerada pelo meu batimento cardíaco). No entanto, e aqui reside o cerne da posição comportamental, a funcionalidade desse evento naquela relação comportamental verbal, dependerá de sua correlação (ou relação de equivalência) com os eventos que constituem a base pública para o uso do conceito. Uma alteração fisiológica qualquer pode ser a ocasião para eu dizer que "estou feliz", mas se não estiver consistentemente correlacionada com os "comportamentos públicos" de felicidade, ela não funcionará como condição para essa descrição. A condição fisiológica que estiver correlacionada com o evento público, por seu turno, pode, inclusive variar substancialmente de uma pessoa para outra, e para um mesmo indivíduo ao longo de sua vida; sua funcionalidade é dada pela relação com o evento público. O que se passa com um indivíduo, do ponto de vista fisiológico, quando está feliz, é muito diferente do que se passa com qualquer outro organismo, até por força da particularidade da constituição anátomo-fisiológica de cada um; é diferente, ainda, do que se passava com ele mesmo, quando ficava feliz alguns anos atrás, também porque desde então se tornou um organismo modificado. $\mathrm{O}$ que há de comum entre felicidade de um indivíduo, hoje, sua própria felicidade anos atrás, e a felicidade dos outros são as relações comportamentais, públicas, que as definem (cf. Tourinho, 2006a, 2006b).

A segunda direção que a abordagem comportamental para a subjetividade assume é a indicação das próprias relações comportamentais constitutivas dos fenômenos a que os conceitos, por exemplo, emocionais, dizem respeito. Desse ponto de vista, um analista do comportamento apontará que esses conceitos constituem respostas verbais emitidas sob controle de fenômenos com graus variáveis de complexidade, relações comportamentais específicas, ou redes de relações interconectadas, exigindo certo cuidado na análise, especialmente quando dela se pretendem derivar estratégias de intervenção. Nos fenômenos emocionais, as relações têm, em geral, um ponto de partida nas emoções básicas, mas, a partir delas, cada história ambiental produz fenômenos muito varia- 
dos. O que esse modelo sugere é que filogênese, ontogênese e cultura funcionam para produzir relações constitutivas dos fenômenos emocionais. Nesse caso, a complexidade está associada à inclusividade. Fenômenos comportamentais mais complexos vão sendo produzidos na medida em que um organismo vai sendo sucessivamente exposto a contingências ontogenéticas e culturais, que, a partir de uma base filogenética, vão dando origem a relações interrelacionadas. Como apontado antes, quando aí se incluem relações verbais, os fenômenos ganham uma nova dimensão, em razão dos processos de transferência de funções entre estímulos, o que, para os psicólogos clínicos, por exemplo, tem se mostrado uma questão central (cf. Tourinho, 2006b).

A interpretação comportamental vira pelo avesso uma série de concepções bem estabelecidas em nossa cultura e impacta muitas de nossas crenças mais ordinárias. Se conhecer é interagir com parcelas do mundo de modos específicos, a partir de uma história ambiental particular, não há um arquivo a ser consultado na mente ou no cérebro; também no caso do autoconhecimento, não há acesso privilegiado a um mundo privado, mas, ao contrário, o autoconhecimento está circunscrito ao que o ambiente social promove de autodiscriminação; se a linguagem é constitutiva do mundo dos sentimentos, nossas "autodescrições de emoções" não representam, mas são parte dos próprios fenômenos emocionais; se o pensar, refletir, calcular etc. são interações do organismo como um todo com o mundo, não estão dentro nem fora do organismo, não estão sequer no organismo.

Conceitos como responsabilidade, autonomia, interioridade, subjetividade etc. todos mereceriam um exame crítico, se partimos da perspectiva oferecida pela análise do comportamento. E há várias razões para que esse exame seja feito, considerando-se, especialmente, o conhecimento científico acumulado sobre as relações comportamentais.

\section{Conclusão}

Este artigo teve o objetivo de oferecer algumas referências históricas sobre o desenvolvimento da Psicologia behaviorista que em larga medida ultrapassam os horizontes sugeridos pela identificação da abordagem com o trabalho de Watson. Como se buscou mostrar, do ponto de vista histórico, algumas realizações anteriores a Watson foram fundamentais para definir o alcance da ciência do comportamento ao longo do século XX, realizações que foram, inclusive, obscurecidas ou negligenciadas pelo próprio Watson.

A vertente do pensamento behaviorista que adquiriu maior vigor no século XX, porque em torno dela se construíram programas de investigação e se organizaram grupos de pesquisa cujo trabalho impactou expressivamente o pensamento psicológico, foi aquela fundamentada no trabalho de Skinner. Essa vertente, hoje constituída como Análise do Comportamento, tem feições, nesta primeira década do século XXI, que também ultrapassam em larga medida o horizonte das investigações realizadas por Skinner. Como argumentado, ainda que o legado intelectual de Skinner constitua a espinha dorsal das realizações da Análise do Comportamento, a disciplina hoje se desenvolve em várias direções (acadêmicas e profissionais) e incorpora contribuições não necessariamente decorrentes das (ou compatíveis com as) idéias skinnerianas. Longe de ser um problema, esta é uma característica que tem permitido à Análise do Comportamento avançar na compreensão de seu objeto de estudos e alcançar maior visibilidade social. Uma característica que tem proporcionado ao pensamento behaviorismo grande sucesso, mesmo quando suas impressões não são claramente identificadas nas práticas e discursos psicológicos para os quais claramente deu uma contribuição fundamental.

Uma apreciação mais completa e precisa dos conjuntos de problemas mencionados ao longo deste artigo certamente requer mais do que o Behaviorismo ou a Análise do Comportamento tem a oferecer, daí também a importância da interlocução com outros sistemas explicativos, na Psicologia e nas demais ciências humanas e sociais. Mas essa necessidade de interlocução não deve obscurecer o fato de que as realizações e os horizontes da ciência do comportamento são largos e de longo alcance, tanto mais quanto arejados por práticas culturais que privilegiem não a mera reprodução de pontos de vista, mas o avanço na abrangência e generalidade de suas descrições.

\section{Referências}

Burgos, J. (2003). Laudable goals, interesting experiments, unintelligible theorizing [Review of the book Relational frame theory]. Behavior and Philosophy, 31, 19-45.

Catania, A. C. (1999). Thorndike's legacy: Learning, selection and the law of effect. Journal of the Experimental Analysis of Behavior, 72, 425-428.

Chance, P. (1999). Thorndike's puzzle boxes and the origins of the experimental analysis of behavior. Journal of the Experimental Analysis of Behavior, 72, 433-440.

Dinsmoor, J. A. (2004). The etymology of basic concepts in the experimental analysis of behavior. Journal of the Experimental Analysis of Behavior, 82, 311-316.

Elias, N. (1994). A sociedade dos indivíduos. Rio de Janeiro, RJ: Jorge Zahar.

Ferster, C. B., \& Skinner, B. F. (1957). Schedules of reinforcement. New York: Appleton-Century-Crofts.

Hall, G. A., \& Chase, P. N. (1991). The relationship between stimulus equivalence and verbal behavior. The Analysis of Verbal Behavior, 9, 107-119.

Harris, M. (2007). Cultural materialism and behavior analysis: Common problems and radical solutions. The Behavior Analyst, 30, 37-47.

Hayes, S. C., Barnes-Holmes, D., \& Roche, B. (2001). Relational frame theory: A post-Skinnerian account of human language and cognition. New York: Kluver Academic.

Hearst, E. (1999). After the puzzle boxes: Thorndike in the 20th century. Journal of the Experimental Analysis of Behavior, $72,441-446$. 
Hilgard, E. R. (1988). Review of B. F. Skinner's The behavior of organisms. Journal of the Experimental Analysis of Behavior, 50(2), 283-286. (Review is reprinted from Psychological Bulletin, 36, 121-125, 1939).

Kantor, J. R. (1922). The nervous system, psychological fact or fiction? Journal of Philosophy, 19, 38-49.

Kantor, J. R. (1970). An analysis of the experimental analysis of behavior (TEAB). Journal of the Experimental Analysis of Behavior, 13, 101-108.

Moore, J. (1987). He's always been there first. The Interbehaviorist, 15, 46-48.

Morris, E. K. (1984). Interbehaviorial psychology and radical behaviorism: Some similarities and differences. The Behavior Analyst, 7, 197-124.

Morris, E. K., Higgins, S. T., \& Bickel, W. K. (1982). The influence of Kantor's interbehaviorial psychology on behavior analysis. The Behavior Analyst, 5, 158-173.

Palmer, D. (2004). Data in search of a principle: A review of Relational frame theory: A post-Skinnerian account of human language and cognition. Journal of the Experimental Analysis of Behavior, 81, 189-204.

Ribeiro, A. F. (1995). Relações de equivalência: Um novo princípio? Temas em Psicologia, 3, 59-64.

Roediger, H. L. (2004). What happened to behaviorism? American Psychological Society (APS) Oberver, 17(3). Retrieved February 20, 2008, from http://www. psychologicalscience.org/observer/getArticle.cfm?id $=1540$

Rorty, R. (1993). Solidariedade ou objetividade. Novos Estudos CEBRAP, 36, 109-121.

Ryle, G. (1984). The concept of mind. Chicago: The University of Chicago Press. (Original work published 1949)

Sério, T. M. A. P. (1990). Um caso na história do método científico: Do reflexo ao operante. Tese de Doutorado nãopublicada, Programa de Estudos Pós-Graduados em Psicologia Social, Pontifícia Universidade Católica de São Paulo, SP.

Sério, T. M. A. P. (1997). A concepção de homem e a busca de autoconhecimento: Onde está o problema? In R. A. Banaco (Ed.), Sobre comportamento e cognição: Vol. 1 (pp. 209-216). Santo André, SP: ARBytes.

Sidman, M. (1994). Equivalence relations and behavior: A research story. Boston: Authors Cooperative.

Skinner, B. F. (1938). The behavior of organism. New York: Appleton-Century-Crofts.

Skinner, B. F. (1945). The operational analysis of psychological terms. Psychological Review, 52, 270-277, 291-294.

Skinner, B. F. (1961). The concept of the reflex in the description of behavior. In B. F. Skinner (Ed.), Cumulative record (enlarged ed., pp. 319-346). New York: Appleton-CenturyCrofts. (Original work published 1931)

Skinner, B. F. (1979). The shaping of a behaviorist. New York: Alfred A. Knopf.

Skinner, B. F. (1987a). Whatever happened to psychology as the science of behavior? American Psychologist, 48, 780786.

Skinner, B. F. (1987b). What is wrong with daily life in the western world? In B. F. Skinner (Ed.), Upon further reflection (pp. 15-31). Englewood Cliffs, NJ: Prentice Hall. (Original work published 1986)

Skinner, B. F. (1990). Can psychology be a science of mind? American Psychologist, 45, 1206-1210.

Skinner, B. F. (1992). Verbal behavior. Acton, MA: Copley. (Original work published 1957)
Skinner, B. F. (1988). The cuckoos. The ABA newsletter, 11(3), 9.

Smith, L. D. (1989). Behaviorism and logical positivism. Stanford, CA: Stanford University Press.

Timberlake, W. (1993). Behavior systems and reinforcement: An integrative approach. Journal of the Experimental Analysis of Behavior, 60, 105-128.

Thorndike, E. L. (1998). Animal intelligence: An experimental study of the associative processes in animals. American Psychologist, 53, 1125-1127. (Original work published 1898)

Thorndike, E. L. (2007). Animal intelligence. Retrieved October 25, 2007, from http://www.dominiopublico.gov.br/download/ texto/ps000178.pdf (Original work published 1911)

Todorov, J. C. (2004). Da aplysia à constituição: Evolução de conceitos na análise do comportamento. Psicologia: Reflexão e Crítica, 17, 151-156.

Tourinho, E. Z. (2004). Behaviorism, interbehaviorism and the boundaries of a science of behavior. European Journal of Behavior Analysis, 5, 15-27.

Tourinho, E. Z. (2006a). On the distinction between private events and the physiology of the organism. The Behavior Analyst Today, 7, 548-559.

Tourinho, E. Z. (2006b). Private stimuli, covert responses and private events: Conceptual remarks. The Behavior Analyst, 29, 13-31.

Tourinho, E. Z. \& Sério, T. M. A. P. (2010). Dimensões contemporâneas da Análise do Comportamento. Em Tourinho, E. Z. \& Luna, S. V. (Orgs.), Análise do comportamento: Investigações históricas, conceituais e aplicadas (pp. 1-13). São Paulo: Roca.

Verplanck, W. S. (1995). Some reflections on Kantor, Kantorians, and Kantor's career. The Interbehaviorist, 23, 6-12.

Watson, J. B. (1970). Behaviorism ( $2^{\text {nd }}$ ed.). New York: W. W. Norton. (Original work published 1930)

Watson, J. B. (1994). Psychology as the behaviorist views it. Psychological Review, 101, 248-253. (Original work published 1913)

Wittgenstein, L. (1988). Philosophical investigations (G. E. M. Anscombe, Trans., $3^{\text {nd }}$ ed.). Oxford, UK: Basil Blackwell. (Original work published 1953) 